\title{
A study on the vertical migration of elements in supergene environment and its application in geochemical anomaly delineation \\ LIJUN YANG
}

Northwest University

Presenting Author: 2252946524@qq.com

In conventional geochemical exploration work, the samples of the same particle size range are used to eliminate the interference of foreign substances. However, the influence of topography on element migration is not considered in the anomaly delineation, and only a unified anomaly lower limit is used to delineate anomalies, which may result in false anomaly or hide some weak mining-induced anomaly information. This research proposes to introduce elevation elements into the processing of geochemical data, divides elevation data groups at equal intervals according to the relative elevation differences, and analyzes the vertical migration characteristics of elements under different landscape conditions. The present study has shown that summarizing the superficial vertical migration of elements can finely divide the geochemical background area, shield the influence of high background values on weak and small anomalies, and have a greater reality in screening, identifying weak and false anomaly information, and guiding actual fieldwork significance.

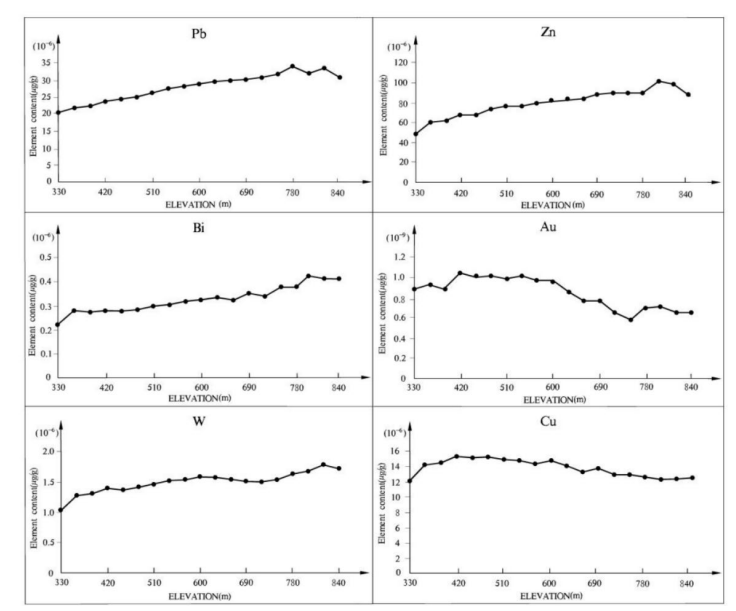

Figure 1 The forest swamp area of the Greater Khingan Mountains average elevation graph (Huma) elements 